\title{
Traffic Optimization and Monitoring Terminals in Ad-Hoc Network through Ant Colony System
}

\author{
Miguel A. Chitaba Díaz, Octavio J. Salcedo-Parra, Brayan S. Reyes-Daza
}

\begin{abstract}
In this article, we will explore the way to optimize the traffic in ad-hoc networks. The approach presented here, uses the study of swarm intelligence, more specifically the ant colony systems (ACS) looking so each of the devices in a given network contribute decentralized to build optimal paths where must transit the information within the network.
\end{abstract}

Keywords: Ad-hoc network, ant colony system, Hamiltonian circuit, stochastic process.

\section{Introduction}

\section{A. 1. Wireless AD-HOC networks}

The ad-hoc network are a type of network that do not need any preexistent infrastructure as routers, wired connections, etc. Instead, each of the nodes or participants in the net- work performs the routing functions and data retransmission. Each node can be asymmetrical according to the quantity of resources that it has, therefore will exist nodes with high throughput and storage, while others have a low capacity or a high occupancy index, whence the selection of the next node to request retransmission or perform processing or storage must have the considered conditions mentioned above.

\section{B. 2. Ant colony optimization}

ACO (ant colony optimization) is a type of optimization de- scribed by Marco Dorigo in (Dorigo, 1992), (Dorigo \& Gambardella, 1997) where he exposes an algorithm that belongs to the category of swarm intelligence, based in the biology of ants and how they manage to find their optimal path from their nest to the source of food without any type of centralized control to rules the best route, in other words, without an orchestration process of their behavior to reach their goal.

\section{Miguel A. Chitiva-Diaz}

Universidad Nacional de Colombia,

Bogotá DC, Colombia

\section{Octavio J. Salcedo-Parra}

Universidad Distrital Francisco José de Caldas Bogotá DC, Colombia

Brayan S. Reyes Daza

Universidad Distrital Francisco José de Caldas Bogotá DC, Colombia

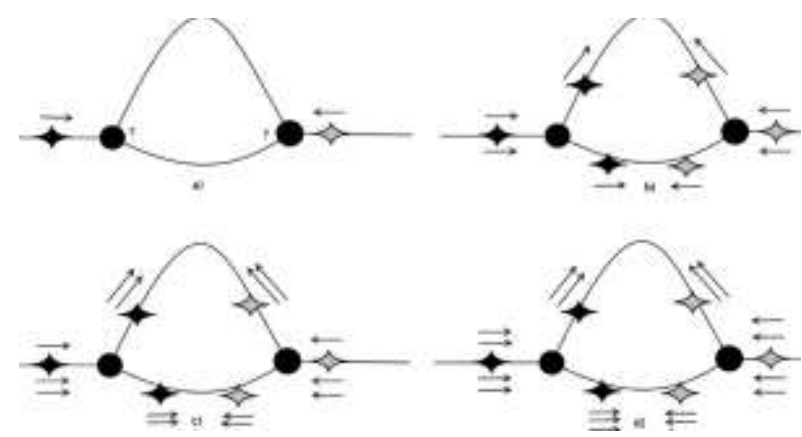

Figure 1. Diagram of the process of successive shortest path.

The main idea behind this stochastic mechanism is the use of a variable named pheromone, a pheromone in nature is a chemical substance secreted by living beings with the purpose of inducting behaviors in other living beings; this is secreted continuously by ants impregnating the path along they transit, the level of this, is represented in the Fig 1 by the amount of arrows in a route segment; to find a bifurcation, each ant will have to choose which of the two paths takes without complete information of distance. 1 a); the procedure is repeated successively as it can be seen in 1 b) and $1 \mathrm{c}$ ). Each one of the ants passing through a segment secretes the same way pheromone, concentrating in the segments of the busiest route. In the other hand in $1 \mathrm{~d}$ ) that in the upper edge is a lower level of pheromone to the lower edge, this is due to a dissipation factor of the pheromone in the environment as it decays faster in places where they have been slow to deposit more pheromone that corresponds to where the path is longer. The key to the process is that the ants will prefer paths with higher concentration of pheromone that will belong to the shortest path sections. Given this statement, the concatenation of multiple segments will offer the shortest route through the segments.

\section{Problem description}

\section{A. Assumptions}

Each node in the network must have an internal monitor which will analyze the rate of use of system resources.

Each node when requested shall return to the applicant the amount of system resources and usage rate of the node, without representing less than the actual amounts.

Are the nodes $k_{i}, k_{j} i \neq j$ nodes belonging to the network, when the node $k_{i}$ requests a service to the node $k_{j}$, $k_{j}$ not denied arbitrarily and unilaterally service to the node $k_{i}$. 
Proc. of the Fourth International Conference on Advances in Computing, Electronics and Communication - ACEC 2016. Copyright (C) Institute of Research Engineers and Doctors. All rights reserved. ISBN: 978-1-63248-113-9 doi: 10.15224/ 978-1-63248-113-9-73

\section{B. Algorithm}

Continuously with a constant period not synchronous with respect to the other nodes, each node in the network will to send a request to the reachable nodes to send the information resources and occupation thereof, such information will be represented by the rate of resource $\lambda^{-1}$.

Once we have the answer of nodes reachable, the response time is calculated $t_{k}$.

Calculate the potency of the signal from node $k$-th in $d B m$ (decibel-miliwatt) $p_{k}$.

Once having the potency and times for reachable nodes, using the transition equation 1

The probability of transition from one node to another will be determined by

$$
P_{k}(r, s)=\left\{\begin{array}{c}
\frac{[\tau(r, s)][\eta(r, s)]^{\beta}[\lambda(r, s)]^{\alpha}}{\sum u \epsilon J_{k}(r)[\tau(u . s)][\eta(u, s)]^{\beta}[\lambda(u, s)]^{\alpha}} \text { if } s \in J_{k}(r) \\
0 \text { otherwise }
\end{array}\right.
$$

Being respectively:

$\tau$ : The level of pheromone to the edge formed by a pair of nodes belonging to the network.

$\eta: \frac{1}{\theta}$ Where $\theta=\left(\frac{Q o S_{t}}{t_{k}}\right)\left(\frac{p_{k}}{Q o S_{p}}\right)^{\phi}$. The first part describes the transmission time, while the second part describes the signal potency.

$Q o S_{t}$ : The acceptable quality of service for time, is a constant $Q o S_{t}>0$ measure in $m s$ to be managed for all nodes and determines the maximum allowable latency for transmission.

$Q o S_{p}$ : Acceptable quality of service for potency, is a constant measure in $\mathrm{dBm}$; determines the minimum allowable potency for transmission $Q o S_{p}>0$.

$\phi$ : It is a proportionality constant which determines the importance of the signal potency relative to the transmission time $\phi$.

\section{$\lambda$ : The inverse of the index resource where $\lambda^{-1}>0$.}

$\beta$ : Proportionality constant that determines the importance of the transmission quality on the medium with respect to the level of pheromone and the resource index.

$\alpha$ : Proportionality constant which determines the importance of the resource quantity compared to the levels of pheromone and transmission quality.

\section{$J_{k}(r)$ : The set of nodes that remain unvisited.}

In (Dorigo, 1992), (Dorigo \& Gambardella, 1997) $\eta$ is associated with the distance between nodes, as path selection criterion, in this problem, the distance is not relevant, but yes the cost of transmission that is associated with the time it takes to transmit from one node to another, and the signal potency, since a signal with a very low power can cause corruption of data and a retransmission will be required. In addition, a new concept is introduced to the original Dorigo's equations, $(\lambda)$ as an ant shall have preference for the node that has the best resources to making the simile that has a more nutritious food for the ant.
Once it have the transition probabilities, one path is selected by a random process considering the probability equation described above; This process will be repeated in subsequent nodes, so that eventually reach the source node providing the map that ran this ant, in other words each ant makes a Hamiltonian circuit. Formally, a path is defined $V=\left\{x_{0}, x_{1}, \ldots, x_{n-1}, x_{n}\right\}$ and $x_{i}=x_{j}$ for $0 \leq i \leq j \leq n$. Moreover, it is said than a circuit $x_{0}, x_{1}, \ldots, x_{n-1}, x_{n}$ is a Hamitonian circuit (Rosen, 2004). Is a Hamilton circuit, if the circuit contains all the nodes of the graph and passing through it only once.

\section{Two Types of Ants}

As explained above, for making a routing algorithm with these conditions, a monitoring mechanism or exploration is needed, so then the tasks of monitoring and minimization tasks are divided into two different kinds of ants.

\section{Ant resource explorer (Type I):}

This type of ant as its name indicates explores the network leading to the starting node the resource map; this map is built in each of the transitions, so that when making a transition from node to node storing the index of resources associated to each of the visited nodes, so to complete the Hamiltonian circuit, ergo when returning to place of origin, ant will bring an association of each node with its own resources index.

This type of ant help us to discover new nodes that have joined to the system and discard those that have also gone in the moment that a node requires a service or resource to another node, you'll know in advance what is the best candidate for this work.

\section{Ant of minimization of the Hamilton circuit (Type II)}

Contrary to the above type of ant, to go through each of the nodes does not collect the resource index. Its function is to collect the node identifiers, the transition time and the signal potency at the time of the transition, in other words the parameter $\eta$ is constructed for each of the transitions, which will subsequently be used for updating the pheromone and consolidate the optimal paths so that with time and after several iterations, converge to an optimal or semi-optimal path. If you look carefully is exactly the same situation of the optimization problem of travelling salesman, best known as TSP (Traveler Salesman Problem), since we must visit each of the nodes of a graph (network) so that the circuit is minimum. Given this important observation can be added as optimization mechanisms parallelized genetic algorithms. A promising approach is described in (Chien \& Chen, 2009).
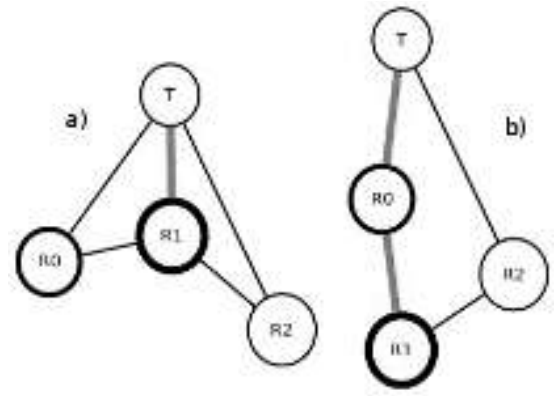

Figure 2. a) A trivial case where all nodes have communication with all members of the network. b) 
Proc. of the Fourth International Conference on Advances in Computing, Electronics and Communication - ACEC 2016. Copyright (C) Institute of Research Engineers and Doctors. All rights reserved. ISBN: 978-1-63248-113-9 doi: 10.15224/ 978-1-63248-113-9-73

Redirection through the nearest node with more resources to achieve the objective.

The reason for the existence of two types of ants does not overload the network with useless traffic that don't adds a new relevant information to the problem as the scout ant resources should be called less frequently, since the network theoretically change little in their resources compared to the state of interconnectivity of the nodes that affect the optimal communication paths; ant type II will be used to not introduce traffic resources as it is futile for the optimization process of the paths.

To achieve a correct assignment of tasks according to the computational resources over the network (choreography) there needs to be some monitoring system to determine which are the devices that are accessed within the network, resources available for that node and time the message reaches and thus possess a resource map and decide before sending the service request, which is the best candidate and the best route for information exchange for meet the resource it needs.

This is no problem if known previously all nodes in the network and we have direct access to them as we can be seen in Figure 2 a), but when a resource is directly inaccessible we have to use an intermediate node 2 b) But how it is known that there is a resource on the network if it is out of range to perceive? That is the reason why need a monitoring system and give us the best route to access that resource.

\section{Update pheromone levels}

As explained above, for making a routing algorithm with these conditions, a monitoring mechanism or exploration is needed, so then the tasks of monitoring and minimization tasks are divided into two d

Local pheromone update: Once an ant completes the circuit, it must register locally updating the route followed pheromone levels by the equation 2 .

$$
\begin{aligned}
\tau(r, s) & \leftarrow(1-\rho) \tau_{i}(r, s)+\rho \tau_{0}(2) \\
& \text { si } \tau_{i}(r, s)<\tau_{\text {min }}, \text { so } \tau_{i}(r, s)=\tau_{\text {min }}
\end{aligned}
$$

$\tau(r, s):$ is the pheromone level to the edge between the nodes $r, s$

$\rho:$ is the parameter of pheromone evaporation.

$\tau_{0}$ : is the initial value of the pheromone, parameter that is studied in depth in (Dorigo \& Gambardella, 1997)

$\tau_{\min }:$ is the minimum possible value of pheromone.

Global pheromone update: Since each node performs its own construction of the optimal path, it can accelerate the process of convergence to the global optimum exchanging information of the individual results of each node; it opts for an elitist approach where only the best ant of the neighborhood may update the pheromone level. The criterion for selecting the best ant in (Dorigo, 1992), (Dorigo \& Gambardella, 1997), (Rosen, 2004), (Chien \& Chen, 2009) is based on the total distance of the Hamiltonian circuit, for our problem will be determined by the index $\eta$ which is a construction that involves time and signal potency.

$$
\begin{gathered}
\tau(r, s) \leftarrow(1-\rho) \tau_{i}(r, s)+\rho \Delta \tau(r, s) \\
\Delta \tau(r, s)=\left\{\begin{array}{c}
\frac{1}{L_{k}} \text { si }(r, s) \in \text { best route group } \\
0 \text { otherwise }
\end{array}\right.
\end{gathered}
$$

When $L_{k}=\min \left\{\sum_{e \in H_{K}} \eta_{e}\right\}$ whit $H_{k}$ as the Hamiltonian path traveled by the ant $k$ and $e$ an edge of $H_{k}$

\section{Planning a route:}

After enough iterations, the solution evolves to an optimal or semi-optimal path. In the event it is desired to acquire a resource not available directly, it is the resource map generated by ants of type I; the selection of the node to delegate an activity will be based on the information resources, as it is presumed that the time required to reach the node will be much lower while processing the request.

In the example of Figure 3; assuming that node 1 does not have the resources to make a productive activity, then once reviewed the resource map choose node 4 as the best candidate for delegate the task, then whit the information Ants type II (the best circuit) it must decide which of the two starting points is the best to transport the request, so it must begin with the nodes numbered 2.3.

The selection of the node will be breaking the circuit in the source and destination nodes, obtaining two different paths (figure 3 der.) which are not necessarily symmetric and one will be shorter than the other, this selection will be determined by the equation 4 .

$$
\min \left\{n_{1}^{\pi}\left(\sum_{e \in H_{1}} \eta_{e}\right), n_{2}^{\pi}\left(\sum_{e \in H_{2}} \eta_{e}\right)\right\}
$$

$n_{i}$ : is the number of jumps performed by the route $i$ - ésimo, and acts as a penalty factor which more jumps it make between the transmission, will be less desirable.

$\pi$ : is a factor of importance of jumping penalty $\pi$.

$H_{j}$ : is the set of edges that form the path $i$ - ésimo

Note that nodes distribute the optimal map, so each frame transported not require transport the path to follow. Each of the nodes know which node is best to carry it on the next step to finally arrive at your destination; the path that it didn't use can serve as a backup in case something goes wrong in the initial transmission. If this support mechanism fails, it should do new inquiry system resources and perform optimization path again.
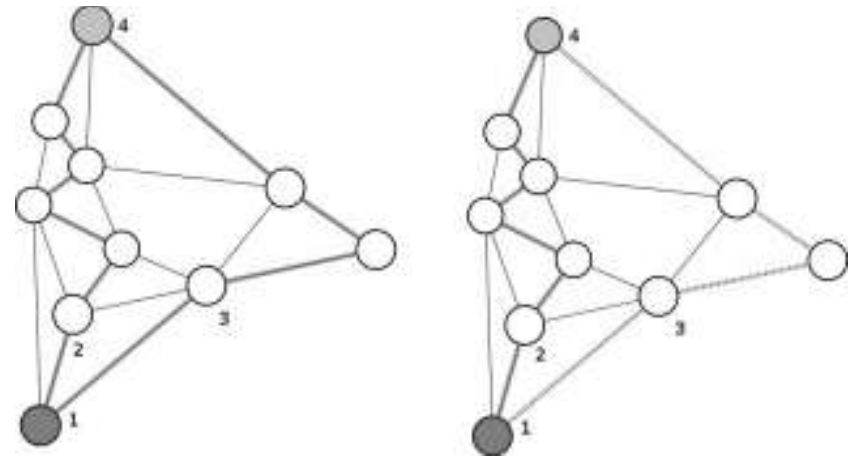

Figure 3. Example of optimal path obtained by the algorithm. 


\section{Algorithmic Complexity}

It is known that the problem of TSP which was reduced the proposed algorithm, is a problem that belongs to the category NP-hard, so falls into the category of the most difficult problems in computation (unless it is proved that $\mathrm{P}$ $=\mathrm{NP})$, so there is no algorithm that solves it in polynomial time, but the proposed evolutionary approach is a cutting meta-heuristics therefore an initial solution evolves over time increasingly providing a better response to the problem, so the focus initially provides an answer that is not optimal and that eventually evolve to a sub-optimal acceptable enough solution (and with some probability to obtain the global optimum because there is always a probability to get to it even in the first iteration given the stochastic nature of the algorithm), hence the algorithm does not ensure the best route, but yes a sufficiently close approximation to the global optimum so that with relatively little effort obtains a form of fast transmission and with a degree of redundancy, it will be described below.

A simple way to understand the complexity of selecting the appropriate order of the edges of the fully connected graph (without cycles to the same vertex) whose vertices are listed is: the first vertex is taken where it checks which has exactly $n-1$ edges that connecting with the other $n-1$ vertex, such that moving the second vertex will have $n-2$ edges since for this case, the edge that connecting the first and second vertex is deducted from the number of possibilities, preserving this behavior for the remaining vertices; as is $n$ the total number of vertex, it follows that the total number of possibilities to select the travel of an edge to a subsequent is $(n-1) !=(n-1)(n-2)(n-$ 3) ... (1), $n$ times

In this way there $(n-1)$ ! paths, but keep in mind that if it has a circuit of the form $a b c$ is equivalent to $c b a$, are therefore indistinguishable paths since they have the same transitions and the same distance, then ignore them, so we have in total $\frac{(n-1) !}{2}$ paths, where the optimal is just one of them all; if $n$ is large enough so intractable is showing that it is a problem of no order polynomial.

This is the worst case, where the graph is fully connected, since one must be chosen (the optimal) between $\frac{(n-1) !}{2}$ available paths, setting the upper limit (if done by brute force, trying every possible ways to find the best), but this case is unrealistic because it implies that all nodes in the network are reachable and it would be trivial to establish the route because it is directly connected, so that in a more realistic case there will be no nodes reachable reducing the number of edges in the graph and in turn reduces the complexity in proportion.
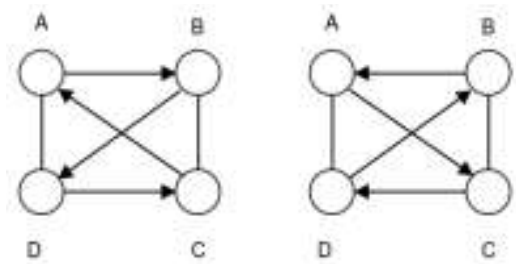

Figure 4. Example of two indistinguishable circuits, even if the route is different these are equivalent.
This complexity is bounded between the worst case and the best case, where the best case is that there is only one and exactly one circuit that interconnects all nodes. Considering the Figure 4, if we eliminate the internal edges: $(a c),(b d)$ there will only be one possible circuit $a b c d$. In other words the number of possible paths is:

$$
\frac{(n-1) !}{2} \geq X \geq 1
$$

A realistic case will be out of both boundary. The above statement does not say much, just that the problem itself has a complexity between constant and factorial, which gives a huge range of features that can describe the behavior, and therefore we will work on the assumption of worst under a pessimist approach to get a good idea ofthe work of the proposed algorithm.

\section{A. Calculation of transmition probabilities:}

Exploration phase: Each scout ant made $n$ transitions to make a Hamiltonian path where each transition will be associated with different transition time where it can show the average time of transition as $t_{c}$, so that the total travel time of ants can be expressed as $t_{c 1}=n t_{c}$.Calculation probability phase: As can be seen from the equation 1 a sum of distinct values done on each of the nodes adjacent and then makes the decision about which node should make the transition, therefore has to total processing time for this action is $t_{p 1}=a$ where $a$ represents the number of adjacent nodes, and will vary from node to node, because each will have a different degree of connectedness. This value is bounded between the best case, that has only one reachable node and the worst case that having $n-1$ reachable nodes, ergo $n-1 \geq a \geq 1$.

\section{B. Calculation of updating local pheromone levels:}

Looks the equation 3 see that it is a simple constant-time operation, but should be made for each of the flown edges, thus a path that loops through each of the nodes have exactly $n-1$ edges, so, will have to upgrade for each ant $n-1$ times, therefore the processing time of this action will $t_{p 2}=n-1$.

\section{Calculating update global pheromone levels:}

Selection phase of the best ant: In this phase, each node receiving a scout ant that has completed the course must do the minimization of the sum for the variable $\eta$ that shown in the equation 3 , this minimization is a simple summation of all $\eta$ for each node route, so $t_{p 3}=n$ but such action must be performed for each of the $n$ nodes, where each node generated a scout ant independently, therefore $t_{p 3}=n^{2}$

Communication phase: In this phase, each of the adjacent nodes share the best solution of the best recorded ant for each node. In this step is necessary to clarify that don't order here, only stored on each node the best exemplary generated. To do this procedure first requires communicating with adjacent nodes or achievable nodes; is a value that differs from node to node, this value is bounded between the maximum granted by variable $Q o S_{t}$ introduced as factor of $\eta$ for the equation 1 and zero, which is the minimum possible value, ergo the transmission time from one node to another is defined by $Q o S_{T} \geq t_{c i} \geq 0$, but given that such transfer should be done with all adjacent nodes, we can see the average transmission time between all adjacent nodes as $t_{c}$. Thus the total transmission time for the $a$ 
Proc. of the Fourth International Conference on Advances in Computing, Electronics and Communication - ACEC 2016. Copyright (C) Institute of Research Engineers and Doctors. All rights reserved. ISBN: 978-1-63248-113-9 doi: 10.15224/ 978-1-63248-113-9-73

adjacent nodes will be determined by $t_{p 4}=a(n-1)$. Processing phase: Once it has the best ants each adjacent node compared to each other; the best one is selected to do the update of pheromone. As has an ant for each adjacent node, it must do a comparisons, while pheromone update process is equal to that seen for the local case, so that the processing time is determined by $t_{p 4}=a(n-1)$

\section{Calculation cutting cycle to find the best path}

As shown in the formula 4 the Hamiltonian path is cut in two, from the start point to the arrival point and a minimization of the variable $\eta$ made, that is presented as a comparison of two minimizations, but is equivalent to the computation of the sum of all variables $\eta$. These are exactly $n-1$, so that $t_{p 5}=n-1$

At this point, it has completed a full cycle of the algorithm, all it takes sum of all previously calculated values, obtaining the following expressions:

$$
\begin{gathered}
T(n)=t_{c 1}+t_{p 1}+t_{p 2}+t_{p 3}+t_{c 2}+t_{p 4}+t_{p 5} \\
T(n)=n \bar{t}_{c}+a+(n-1)+n^{2}+a \bar{t}_{c}+a(n-1)+ \\
(n-1)
\end{gathered}
$$

Insomuch as $a$ represents the number of adjacent nodes, and as defined above $n-1 \geq a \geq 1$. Taking the pessimistic approach in order to find the upper bound of the algorithm, as defined at the beginning of this analysis will take $a=n-1$. On the other hand $\overline{t_{c}}$ the average time is a constant $Q o S_{T} \geq t_{c} \geq 0$, and again looking for the worst case we take $t_{c}=Q o S_{T}$, but ultimately the value is a constant, which can be eliminated from the analysis, obtaining:

$$
\begin{gathered}
T(n)=n+(n-1)+(n-1)+n^{2}+(n-1) \\
+(n-1)(n-1)+(n-1) \\
T(n)=n^{2}+(n-1)^{2}+5 n-4 \\
T(n)=0\left(n^{2}\right)
\end{gathered}
$$

That is a complete cycle which analyzes $n$ possible solutions, has a complexity of $O\left(n^{2}\right)$. Each of these cycles has a probability of finding the global optimum of $P(X)=$ $\frac{n}{\frac{(n-1) !}{2}}=\frac{2 n}{(n-1) !}$. As you can observe is unlikely, but there is still some chance that in just a cycle the algorithm can find the global optimum of a problem in order $O(n !)$ with order processing of $O\left(n^{2}\right)$. The most interesting aspect is that it has a method of polynomial order to be found near the optimal paths in an efficient manner, this solution improved in each of the subsequent iterations, as shown in (Feng, 2010) in some experiments in just 50 iterations can achieve sub-optimal solutions with an error rate from the relatively small global optimum and that the margin of error decreases progressively in subsequent iterations.

\section{Conclusions}

The proposed algorithm can be used for the purposes of resource discovery in ad-hoc networks and optimization of route for the data transmission between different terminals, representing an option to build on this foundation different communication mechanisms for such networks, which are expected in the coming decades increasingly implementation and adoption by the consumer sector.
Relaxing the algorithm and using only ants of type I can be an efficient mechanism to monitoring terminals as sensors of various kinds; moreover using other evolutionary strategies as genetic algorithms can significantly improve the convergence time in the optimal routes.

With the new variables introduced to the model already developed by Dorigo, further studies should be made in the forms of selection constants to have the right balance between the importance of the pheromone with respect to the rate of resource, transmission time and potency of signal between nodes.

\section{References}

[1] Chien, C. Y. \& Chen, S. M. (2009). A New Method for Handling the Traveling Salesman Problem Based On Parallelized Genetic Ant Colony Systems, Proceedings of the Eight International Conference on Machine Learning and Cybernetics, Baoding. p. 2828 - 2833.

[2] Dorigo, M. (1992). Optimization, Learning and Nature Algorithm, Italia, Politecnico di Milano. Dipartimento di Electtonica,

[3] Dorigo, M. \& Gambardella, L.M. (1997). Ant colonies for the traveling salesman problem, Biosystems. p. 73-81

[4] Dorigo, M. \& Gambardella, L.M. (1997. Ant colony system: A cooperative learning approach to the traveling salesman problem, IEEE Transactions on Evolutionary Computation, p. 53-66.

[5] Feng Y. (2010). Ant colony for the TSP. Master Thesis, Högskolan Dalarna. Computer Engineering. Dalarna University College. Falun, Suecia.

[6] Rosen, K. H. (2004). Matemática Discreta y sus Aplicaciones, Mc Graw Hill, Interamericana De España: S.A. Mcgraw-Hill.

About Author (s):

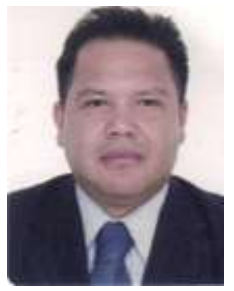

Ing. Octavio José Salcedo Parra: Systems Engineer, Universidad Autónoma de Colombia. Magister in Teleinformatics, District University, "Francisco José de Caldas". Master in Economics, Universidad de los Andes.

Ing. Brayan Steven Reyes Daza: Systems Engineer, Francisco José de Caldas District University, Monitor of the "Intelligent Internet" Research Group.

Ing. Miguel A. Chitaba Díaz: Systems Engineer, National University of Colombia, Researcher of the "Intelligent Internet" Research Group. 